\title{
An Interdisciplinary Oral Health Program for Children in Kindergartens of Northern Italy
}

\section{A Orlando ${ }^{1,2}$, MW Arisido ${ }^{3}$, S Brioschi ${ }^{1}$, L Maccà ${ }^{1}$, S Graci ${ }^{1}$, MC Panzeri ${ }^{1}$, F Mecatti ${ }^{3}$, P Palestini ${ }^{1,2}$ and E Cazzaniga ${ }^{1,2 *}$}

${ }^{1}$ School of Medicine and Surgery, University of Milano, Bicocca, Italy

${ }^{2}$ Bicocca Center of Science and Technology for FOOD, University of Milano, Bicocca,

Italy

${ }^{3}$ Department of Sociology and Social Research, University of Milano, Bicocca, Italy

*Corresponding Author: E Cazzaniga, School of Medicine and Surgery, University of

Milano, Bicocca, Italy.
Received: April 26, 2021

Published: May 20, 2021

(C) All rights are reserved by E Cazzaniga., et al.

\begin{abstract}
Background: The aim of the study was to investigate if a parents' training program on nutritional and oral health behaviours held by health professionals can influence children habits in a sample of kindergartens in Northern Italy.

Methods: The study designed was a longitudinal study. The study population were children aged 6-36 months attending four kindergartens. Parents were invited to participate to a training meeting and to fill out a self-administered questionnaire at baseline and after 3 months from the meeting. The questionnaire included information on socio-demographics about parents, oral hygiene habits of parents and child, and eating habits of child. Wald test was used to analyse data collected.

Results: After the training program, almost all parents could use a toothbrush suitable for kids (from 91\% at the baseline to $99 \%$ after the 3-months). The analysis shows that the given training significantly increased the number of children who use toothpaste from $86 \%$ at baseline (95\%CI: 85\% - 88\%) to $96 \%$ (95\%CI: $94 \%-98 \%$ ), in particular a fluoride toothpaste (59\% to $80 \%$ ). The intervention study showed a positive impact on the number of pupils who wash their tooth more than twice per week and on the timing of oral hygiene as both night and morning time proportion increased. Regarding the feeding habits the given consultation resulted in a statistically significant increase the morning snack from 94\% (95\%CI: 92\% - 96\%) to 97\% (95\%CI: 94\% - 99\%). Another promising effect is the decrease from $47 \%$ at baseline (95\%CI: $46 \%$ - 49\%) to $42 \%$ after the study (95\%CI: $41 \%$ - $43 \%$ ) of the bad habit of having a snack after dinner.

Conclusion: Results of our study have shown that a parents training intervention can have good results on the oral hygiene and eating habits of children of this age group. To underline the importance of several professional figures who work together with a common purpose.
\end{abstract}

Keywords: Children; Oral Health; Dental Caries; Educational Programme; Nutrition; Prevention

\section{Introduction}

Dental caries (tooth decay) is one of the most prevalent infectious diseases among children and is caused by the activity of the common oral micro flora [1].
Caries can be considered as a lifestyle disease that can largely be prevented by maintaining strict but simple nutritional habits (e.g. low sugar intake, limited night-time bottle-feeding and no sharing

Citation: E Cazzaniga., et al. "An Interdisciplinary Oral Health Program for Children in Kindergartens of Northern Italy". Acta Scientific Nutritional Health 5.6 (2021): 36-42. 
of eating utensils) and by applying appropriate oral health behaviours (e.g. regular dental visits, tooth brushing and using fluoridated toothpaste) $[1,2]$.

Recent epidemiological surveys indicate a reduction in the prevalence of caries in Italy which is in line with the trend observed in industrialized countries $[3,4]$. Nobile et colleagues reported that $19 \%$ of the children aged 36 - 71 months attending kindergartens had experienced early childhood caries (ECC) [5].

Given the existence of a correlation between caries of deciduous dentition and the prevalence of caries in permanent dentition [6], we think it is necessary to intervene from early childhood [4] to develop good dental hygiene habits.

Another important aspect is the feeding of the first years of life. It is, indeed, proven that the food choices and frequency are important risk factor for caries in children [7]. Early feeding habits, involve a number of simultaneous and interrelated behaviours, such as breastfeeding, bottle-feeding and the introduction of complementary foods [8]. Moreover, it is demonstrated that some feeding behaviours established in the first three years of the child's life may be maintained in later years, potentially impacting in oral health over the life course [9]. In particular, specific feeding practices, initiated earlier than 12 months, have been linked to caries: addition of sweeteners and sweet snack consumption before 6 months [10].

Parents can be considered the most important actor in caries prevention especially for children under the age of 10 . The parents' willingness to invest time and effort to do so will depend, among other things, on their knowledge and their perceptions of the effectiveness of preventing caries by maintaining proper oral hygiene $[1,11]$.

Moreover, schools can provide an ideal setting for nutrition interventions as they serve as a focal point to involve families and educators, to implement effective and sustainable aid for children. Studies have shown that nutrition education programs have an effect on improving good eating habits, it is important to underline that the parental involvement can increase children's nutritional behaviour [12].

\section{Aim of the Study}

The aim of this study was to investigate if a parents' training program on nutritional and oral health behaviours held by health professionals can influence children habits.

\section{Methods}

Study design

The Ethics Committee of XXXX approved this longitudinal study (protocol 298 03/04/2017). The study was conducted with the informed consent of all caregivers and in full accordance with the ethical requirements of the World Medical Association Declaration of Helsinki (2008).

The study was based on kindergartens located in the northern part of Italy, with four nursery locally named as 'Bambini Bicocca' (Milan), 'Le Ciliegine' (Monza), 'La Piccola Tribù' (Grignano, BG), 'Maria Bambina' (Lissone, MB).

\section{Prevention of caries program}

Parents and kindergartner educators were counselled on general and oral health, toothbrushes and Typodont were used for the illustration of correct oral hygiene manoeuvres. It was advised that tooth brushing should be started at time of eruption of the first tooth and should be undertaken twice a day after meals with an age appropriate toothbrush and a pea-sized layer of fluoride toothpaste (1000 ppm NaF or amine fluoride) $[13,14]$. Parents should schedule a dental visit in the first year of life of their children followed by regular dental care with annual dental examinations. Every family received a folder with brief information materials and a toothbrush and fluoride toothpaste were provided as incentives.

The main nutritional aspects treated during the meeting were the importance of taking five meals per day, in particular having a morning and afternoon snack, the role of fruit and vegetables daily consumption, the importance of drinking water according to the World Health Organization guidelines (WHO guidelines) [15]. Particular attention was given to instruct parents to prefer slowly absorbed sugars and drastically reduce the intake of free sugars with the diet. The child diet should include fibre-rich foods and low in simple sugar. Water should be preferred over any sweetened drink in order to intake less than $10 \%$ of energy from free sugars as recommended by WHO [16].

\section{Questionnaire and data collection}

After the signing of the informed consent, all parents were invited to fill out a questionnaire (baseline, T1). Next, there was a parents' training meeting in which correct oral hygiene manoeuvres 
and correct eating habits were explained. The meeting were realized by health professionals (dental hygienists and nutritionists) of University of Milano-Bicocca. After 3 months (T3) from the training meeting, the same questionnaire was given to evaluate the general improvement of family habits.

The questionnaire completed by parents included information on: socio-demographics status about parents (sex, age, education level) and child (sex, age, gender, date and place of birth), oral hygiene habits of parents and child (frequency of tooth brushing at the moment of the interview, supervision of toothbrushing, use of toothpaste, exposure to fluoride), nutritional habits (frequency of the snack during the day, type of food intake).

\section{Statistical analysis}

A longitudinal study was adopted, with data collection at baseline (T1) and after 3-month (T3).

Descriptive statistics and plots were used to summarize the data. The trend/evolution of the main variables such as quantity and timing of tooth washing, feeding habit during the follow-up (from T1 to T3) was evaluated using a linear mixed-effects model with a random intercept and random slope by treating time as a continuous variable. These response variables were assumed to follow a binomial distribution in the linear mixed effects model.

\section{Results}

The number of subjects interviewed at baseline $\mathrm{T} 1$ were $\mathrm{N}=$ 103 , and after the training intervention 68 of them provided complete data. The average age of the participating children at baseline was 2.249 ( 2 years and 3 months) (SD $=0.91$ years) with the youngest 0.55 ( 6 months) and the oldest 5 years. With regards to the parents who received the training, the average age at baseline was 36.89 (SD = 4.78) with the youngest 18.54 and the oldest 49.90 years. $72.5 \%$ of the parents completed university degree qualification, while $26.5 \%$ and $1 \%$ completed high school and middle school (higher than elementary), respectively. As for parents dental cleaning habit, $2 \%$ clean once a day, $56.1 \%$ clean twice and $41.9 \%$ three times per day.

Table 1 reports results with regard to the impact of the given study intervention on a set of 8 variables used to describe oral hygiene. These variables include, toothbrush for kids, use of fluoride toothpaste, the habit of toothpaste use, number of dental wash per

\begin{tabular}{|l|c|c|c|}
\hline & Baseline & $\begin{array}{c}\text { After 3-month } \\
\text { follow-up }\end{array}$ & \\
\hline & $\begin{array}{c}\text { Percentage } \\
\text { (95\% CI) }\end{array}$ & $\begin{array}{c}\text { Percentage } \\
\text { (95\% CI) }\end{array}$ & P-value \\
\hline Autonomy & $33(27,40)$ & $30(28,32)$ & 0.636 \\
\hline Toothbrush for kids & $91(86,96)$ & $99(98,100)$ & 0.00826 \\
\hline Use of Toothpaste & $86(85,88)$ & $96(94,98)$ & 0.0494 \\
\hline $\begin{array}{l}\text { Use of fluoride } \\
\text { toothpaste }\end{array}$ & $59(50,69)$ & $80(79,83)$ & 0.0275 \\
\hline $\begin{array}{l}\text { Number of wash per } \\
\text { week }\end{array}$ & $46(44,64)$ & $59(57,61)$ & 0.1250 \\
\hline Night wash & $80(78,82)$ & $90(88,91)$ & 0.1310 \\
\hline Morning wash & $57(54,59)$ & $68(65,70)$ & 0.2152 \\
\hline Dental visit & $12(10,14)$ & $14(12,16)$ & 0.8737 \\
\hline Feeding habit & \multicolumn{3}{|l}{} \\
\hline Morning snack & $94(92,96)$ & $97(94,99)$ & 0.0038 \\
\hline Afternoon snack & $2.8(2.7,3)$ & $3.4(3.0,3.5)$ & 0.0256 \\
\hline Night snack & $47(46,49)$ & $42(41,43)$ & 0.6015 \\
\hline
\end{tabular}

Table 1: Impact of the study intervention.

week (indicated as greater than or less than twice per week), time of wash and various snack time and dental visit.

We noticed that after the training program, almost all children were able to use an appropriate toothbrush suitable for the kids. This was confirmed by the increase from $91 \%$ at the baseline to $99 \%$ after 3-month with a strongly significant $\mathrm{P}$-value = 0.0082 . The analysis of the data also shows that the given training significantly increased the number of children who use toothpaste from $86 \%$ at baseline (95\%CI: 85\% - 88\%) to $96 \%$ (95\%CI: $94 \%-98 \%$ ) with P-value $=0.0494$. The use of fluoride toothpaste was significantly influenced by the training intervention as the baseline measure of $59 \%$ proportion was increased to $80 \%$ with $\mathrm{P}$-value $=0.0275$. The intervention study increased the number of pupils who wash their tooth more than twice per week from $46 \%$ at baseline (95\%CI: $44 \%-64 \%$ ) to 59\% after 3-month (95\%CI: $57 \%-61 \%$ ), although the increase was not statistically significant (P-value $=0.1250)$. Similarly, the intervention showed a positive impact on the timing of oral hygiene as both night and morning time proportion increased after the 3-month. The trend of these variables are also reported in Figure 1. 


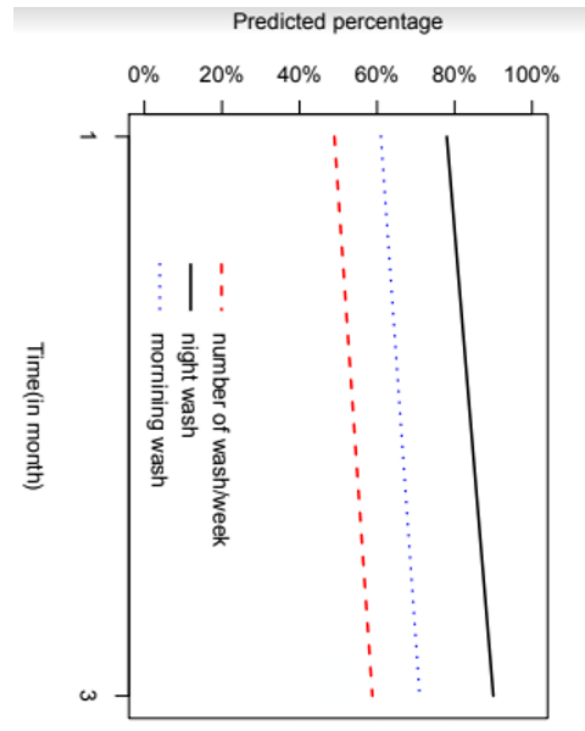

Figure 1: Predicted proportion of pupils from the longitudinal models regarding their oral hygiene.

Regarding the feeding habits the given consultation resulted in a statistically significant increase regarding the importance of morning snack from $94 \%$ at baseline (95\%CI: 92\% - 96\%) to $97 \%$ after 3-month (95\%CI: 94\% - 99\%) with P-value $=0.0038$. The number of pupils favouring afternoon snack is generally low, but the intervention training significantly increased from $2.8 \%$ to $3.4 \%$ with P-value $=0.0256$. Another promising effect of the education is that the decrease from $47 \%$ at baseline (95\%CI: $46 \%$ - 49\%) to $42 \%$ after the study (95\%CI: $41 \%-43 \%$, P-value $=0.6015$ ) of the bad habit of having a snack after dinner.

Figure 2 shows the box plot of the feeding behaviour, in which the study intervention generally influenced the type of food intake. Specifically, the frequency of meat and fish are greatly increased after three months. Cheese consumption has shown a slight increase after the study. While none of the foods has shown a decreasing trend, pizza and egg remained relatively stable.

\section{Discussion and Conclusion}

One of the main results achieved by our project was to increase the number of children using toothbrush and toothpaste specific

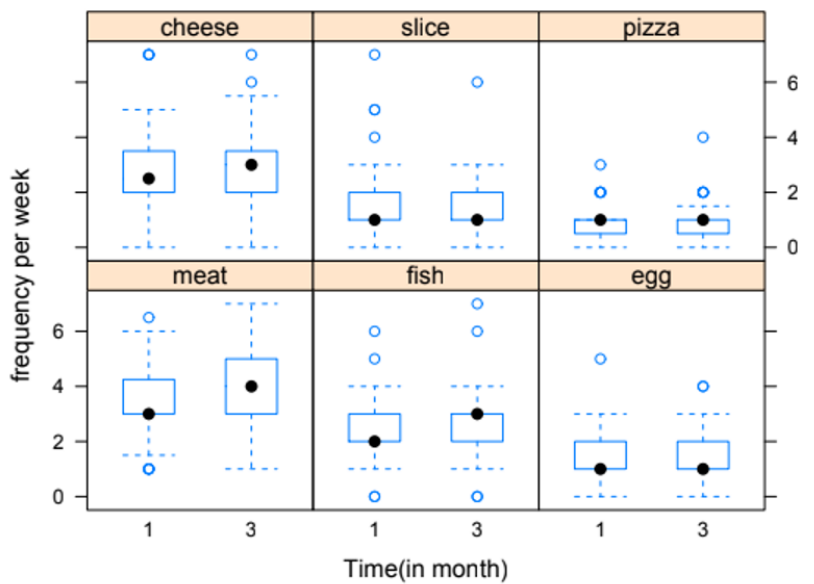

Figure 2: The box plots summarizing the feeding habits before and after the intervention study.

for the age group. Regarding toothpaste, we had a statistically significant increase in children using one containing fluorine. The use of fluorine is the principal factor that reduces the prevalence of caries $[4,17]$. Fluoride is known to prevent caries by inhibiting demineralization enhancing remineralization and inhibiting bacterial growth [18]. In fact, the national guidelines for the promotion of oral health and the prevention of oral pathologies in developmental age (National guidelines 2013) indicate that from 6 months to 6 years of age (age group in which our children fall), fluoroprophylaxis can be carried out through the use of a toothpaste containing at least $1000 \mathrm{ppm}$ of fluorine, 2 times a day, in a pea-size dose.

The National Guidelines for the Promotion of Oral Health and the Prevention of Oral Diseases in Developmental Age (National guidelines 2008) also emphasize that since the eruption of the first deciduous tooth, the teeth must be thoroughly cleaned with gauze or rubber finger. The use of the toothbrush with a small-head soft brush should be started as soon as possible [19] to familiarize the child with it.

The analysis of the data also report an increasing trend in the number of daily washes, both in the evening and in the morning. The removal of soft deposits from oral surfaces is important for the maintenance of dento-periodontal health [14]. However, there 
is only a weak relationship between the frequency of brushing and the reduction in the incidence of caries: it is, in fact, difficult to distinguish between the preventive effect given by the mechanical removal of the plaque and the effect offered by the fluorine contained in the toothpaste $[5,14]$. The Italian guidelines report that the correct brushing of the teeth, at least twice a day, prevents gingivitis; there is scientific evidence that the correct oral hygiene habits must be acquired during childhood [19], to be strengthened later during adolescence. Our data highlight another important fact, our educational intervention has reduced the number of children who brush their teeth independently without any parental support. In fact, it is strongly suggested that oral hygiene for children from 0 to 3 years should be complete parents responsibility [5,20,21]; also it is important that parents set a good example: the child who sees parents brushing their teeth daily several times a day is spontaneously led to imitate them $[1,2,13]$.

Unfortunately, our data report that there was no increase in the number of children who made their first dental visit, a topic that we had instead emphasized during the parents' meeting. In fact, it is known that the first dental visit should be made around 18/24 months regardless of the presence or absence of dental problems $[4,22]$. Moreover that patients under 6 years of age with low risk of developing caries should carry out check-ups every 12 months in case the dentist evaluates a greater risk, the visits will be more frequent [14].

As for the development of caries, it must be emphasized that there are various carbohydrates that can be effectively fermented by bacteria. In addition to sucrose, in order of carcinogenicity, there are glucose, maltose, fructose and lactose [23]. Therefore, the intake of drinks and foods containing simple carbohydrates is not recommended outside main meals [17,19,24]. In this regard, we have observed only a slight trend in the reduction of children who snack at night. The use of sweetened pacifier and the non-nutritional use of the bottle containing sugary drinks must be strongly discouraged $[13,14]$ especially at night, when the salivary flow is greatly reduced. In this regard, during the meetings, we suggested to the parents to continue, at least for a certain period of time, bottle feeding at night, if this was used to aid sleep, but only with water [13].

School-based educational programs held by health professionals may offer the best opportunities for implementing useful and sustainable interventions that are effective in both children and adolescents (Orlando, 2019). Contento defined nutrition education as "any combination of educational strategies, accompanied by environmental supports, designed to facilitate voluntary adoption of food choices and other food- and nutrition-related behaviors conducive to health and well-being" [25]. For successful nutrition education interventions it is important to study the specific characteristics of effective nutrition education programs and to identify the aspects of nutrition education that are essential for the age group considered [26]. We hypothesized that the success of the food education intervention is due to the strategy adopted: engaging neoparents, generally more likely to improve, organizing face-to-face sessions in order to use appropriate and effective communication, identifying specific behaviors to be modified, and the support of teachers.

Our project has ambitious goals for the future such as involving a larger number of individuals, expanding the geographical range of interest and structuring increasingly appropriate meetings.

In conclusion, the results of our study suggest that most of the known modifiable factors concerning eating habits and oral hygiene are still widespread in the population. Moreover, importance emerged of a teamwork composed of nutritionists and dental hygienists who work together in order to achieve the same goals, the health of the child.

\section{Acknowledgments}

We thank the educators of kindergartens involved for the excellent cooperation.

\section{Ethics Approval Statement}

This study was conducted in full accordance with the ethical requirements of the World Medical Association Declaration of Helsinki (2008). The Ethics Committee of University of Milano-Bicocca approved this study (protocol 298 03/04/2017).

\section{Conflict of Interests Disclosure}

The authors declare that they have no conflict of interest.

\section{Funding Statement}

The study received financial support by Lions Club Ponte S. Pietro Isola (Bergamo, Italy). The funder had no role in study design, 
data collection and analysis, decision to publish or preparation of the manuscript.

\section{Patient Consent Statement}

This study was conducted with the informed consent of all caregivers.

\section{Data Availability Statement}

The datasets used and/or analysed during the current study available from the corresponding author on reasonable request.

\section{Bibliography}

1. Vermaire JH and van Exel N. "Parental attitudes towards oral health and caries-risk in their children". International Journal of Dental Hygiene 16.2 (2018): 241-248.

2. Baggio S., et al. "Early childhood caries in Switzerland: a marker of social inequalities". BMC Oral Health 15 (2015): 82.

3. Campus G., et al. "Changing trend of caries from 1989 to 2004 among 12-year old Sardinian children". BMC Public Health 7 (2007): 28.

4. Ferrazzano GF, et al. "Relationship Between Social and Behavioural Factors and Caries Experience in Schoolchildren in Italy". Oral Health and Preventive Dentistry 14.1 (2016): 55-61.

5. Nobile CG., et al. "Pattern and severity of early childhood caries in Southern Italy: a preschool-based cross-sectional study". BMC Public Health 14 (2014): 206.

6. Jordan AR., et al. "Early Childhood Caries and Caries Experience in Permanent Dentition: A 15-year Cohort Study". Swiss Dental Journal 126.2 (2016): 114-119.

7. Ccahuana-Vásquez RA., et al. "Effect of frequency of sucrose exposure on dental biofilm composition and enamel demineralization in the presence of fluoride". Caries Research 41.1 (2007): 9-15.

8. Ruel MT and Menon P. "Child feeding practices are associated with child nutritional status in Latin America: innovative uses of the demographic and health surveys". Journal of Nutrition 132.6 (2002): 1180-1187.

9. Fall CH., et al. "Infant-feeding patterns and cardiovascular risk factors in young adulthood: data from five cohorts in low- and middle-income countries". International Journal of Epidemiology 40.1 (2011): 47-62.

10. Thitasomakul S., et al. "Risks for early childhood caries analyzed by negative binomial models". Journal of Dental Research 88 (2009): 137-141.

11. Schwarzer R., et al. "Stage-matched minimal interventions to enhance physical activity in Chinese adolescents". Journal of Adolescent Health 47.6 (2010): 533-539.

12. Orlando A., et al. "Can Nutrition Education in Primary Schools be an Efficient Way to Prevent Obesity in Adult Life? A Review of the Literature". Acta Scientific Nutritional Health 3.9 (2019).

13. National guidelines for the promotion of oral health and the prevention of oral diseases in developmental age, Ministero del Lavoro, della Salute e delle Politiche Sociali, Italia (2008).

14. National guidelines for the promotion of oral health and the prevention of oral diseases in developmental age, Update, Ministero della Salute, Italia (2013).

15. World Health Organization guidelines (2020).

16. Paglia L. "WHO: healthy diet to prevent chronic diseases and caries". European Journal of Paediatric Dentistry 19.1 (2018): 5.

17. Palacios C., et al. "Nutrition and health: guidelines for dental practitioners". Oral Disease 15.6 (2009): 369-381.

18. American Academy of Pediatrics. "Maintaining and improving oral health of young children". Pediatrics 134 (2014): 12241228.

19. Wagner Y., et al. "Evaluation of a regional German interdisciplinary oral health programme for children from birth to 5 years of age". Clinical Oral Investigation 21.1 (2017): 225-235.

20. Castilho AR., et al. "Influence of family environment on children's oral health: a systematic review". Journal of Pediatrics 89.2 (2013): 116-123.

21. Corrêa-Faria P., et al. "Incidence of dental caries in primary dentition and risk factors: a longitudinal study". Brazilian Oral Research 30.1 (2016): S1806-83242016000100254. 
22. Clinical recommendations in odontostomatology, Ministero Della Salute, settembre (2017).

23. Karjalainen S. "Eating patterns, diet and dental caries". Dental Update 34.5 (2007): 295-298.

24. Ugolini A., et al. "Trends in Early Childhood Caries: An Italian Perspective". Oral Health and Preventive Dentistry 16.1 (2018): 87-92.

25. Contento IR. "Nutrition Education: Linking Research, Theory, and Practic. Burlington, MA Jones and Bartlett Learning". 3rd ed (2016).

26. Murimi MW., et al. "Factors that contribute to effective nutrition education interventions in children: a systematic review". Nutritional Review 76.8 (2018): 553-580.

Volume 5 Issue 6 June 2021

(C) All rights are reserved by E Cazzaniga., et al. 